\title{
Les technologies de l'information et de la communication en pédagogie universitaire et en formation à la profession enseignante : mythes et réalités Information and Communication Technologies in University Pedagogy and Teacher Training: Myths and Realities Las tecnologías de la información y de la comunicación en pedagogía universitaria y en formación a la profesión de maestro : mitos y realidades
}

François Larose, Robert David, Sylvain Lafrance et Judith Cantin

Volume 27, numéro 1, printemps 1999

Perspectives d'avenir en éducation

URI : https://id.erudit.org/iderudit/1080486ar

DOI : https://doi.org/10.7202/1080486ar

\section{Aller au sommaire du numéro}

\section{Éditeur(s)}

Association canadienne d'éducation de langue française

\section{ISSN}

0849-1089 (imprimé)

1916-8659 (numérique)

Découvrir la revue

\section{Citer cet article}

Larose, F., David, R., Lafrance, S. \& Cantin, J. (1999). Les technologies de l'information et de la communication en pédagogie universitaire et en formation à la profession enseignante : mythes et réalités. Éducation et francophonie, 27(1), 100-127. https://doi.org/10.7202/1080486ar
Résumé de l'article

Dans cet article, nous présentons dans un premier temps une revue de la littérature traitant des enjeux épistémologiques qui sous-tendent l'intégration des technologies de l'information et de la communication en pédagogie universitaire. Nous identifions ensuite l'impact de ces enjeux sur le recours aux TIC en formation à la profession enseignante. Enfin, nous présentons les résultats d'une enquête réalisée auprès du professorat de l'Université de Sherbrooke au regard du profil d'alphabétisation informatique, d'attitudes par rapport à l'informatique et des pratiques d'intégration des TIC en enseignement au premier cycle. Les résultats sont examinés à la lumière des conclusions que nous tirons de la revue de la littérature scientifique anglosaxonne.
Tous droits réservés (C) Association canadienne d'éducation de langue française, 1999
Ce document est protégé par la loi sur le droit d'auteur. L'utilisation des services d'Érudit (y compris la reproduction) est assujettie à sa politique d'utilisation que vous pouvez consulter en ligne. 


\section{Les technologies de I'information et de la communication en pédagogie universitaire et en formation à la profession enseignante: mythes et réalités}

\section{François LAROSE}

Université de Sherbrooke, Québec, Canada

\section{Robert DAVID}

Université de Sherbrooke, Québec, Canada

\section{Sylvain LAFRANCE}

Université de Sherbrooke, Québec, Canada

Judith CANTIN

Université de Sherbrooke, Québec, Canada

\section{RÉSUMÉ}

Dans cet article, nous présentons dans un premier temps une revue de la littérature traitant des enjeux épistémologiques qui sous-tendent l'intégration des technologies de l'information et de la communication en pédagogie universitaire. Nous identifions ensuite l'impact de ces enjeux sur le recours aux TIC en formation à la 
profession enseignante. Enfin, nous présentons les résultats d'une enquête réalisée auprès du professorat de l'Université de Sherbrooke au regard du profil d'alphabétisation informatique, d'attitudes par rapport à l'informatique et des pratiques d'intégration des TIC en enseignement au premier cycle. Les résultats sont examinés à la lumière des conclusions que nous tirons de la revue de la littérature scientifique anglosaxonne.

\section{ABSTRACT}

\section{Information and Communication Technologies in University Pedagogy and Teacher Training: Myths and Realities}

François LAROSE, Robert DAVID, Sylvain LAFRANCE, Judith CANTIN

University of Sherbrooke, Québec, Canada

The author first reviews the scholarly literature on the epistemological issues underpinning the integration of communications and information technologies into university teaching. He then identifies the impact of these issues on the use of CITs in teacher training. The paper presents the results of a study carried out with the teaching staff at Université de Sherbrooke concerning computer literacy profiles, attitudes towards computer technology and the methods used to integrate CITs into teaching at the undergraduate level. These results are examined in the light of conclusions based on a review of English-speaking scientific literature.

\section{RESUMEN}

\section{Las tecnologías de la información y de la comunicación en pedagogía universitaria y en formación a la profesión de maestro: mitos y realidades}

François LAROSE, Robert DAVID, Sylvain LAFRANCE, Judith CANTIN

Universidad de Sherbrooke, Québec, Canada

En este artículo presentamos un examen de la literatura que aborda los desafíos epistemológicos sobre los que se funda la integración de la tecnología de la información y de la comunicación en la pedagogía universitaria. En seguida abordamos el impacto que dichos desafíos ejercen sobre la utilización de la TIC en la formación de los maestros. Finalmente presentamos los resultados de una encuesta realizada entre el profesorado de la Universidad de Sherbrooke sobre el perfil de la alfabetización informática, las actitudes con relación a la informática y las prácticas de integración de las TIC en la enseñanza en el primer ciclo universitario. Se examinan los resultados a la luz de las conclusiones que obtuvimos en el examen de la literatura científica anglosajona. 


\section{Introduction}

Depuis 1996, l'État a conçu une série de mesures destinées à assurer que la formation à la profession enseignante intègre le développement de conditions favorisant l'acquisition de compétences minimales en matière d'utilisation pédagogique des technologies de l'information et de la communication (Gouvernement du Québec, 1996a, 1996b). Concrètement, le ministère de l'Éducation a octroyé plusieurs millions de dollars aux facultés d'éducation du Québec afin qu'elles puissent se doter d'infrastructures et d'équipements permettant d'assurer la formation des étudiantes et des étudiants dans le domaine. Du même souffle, l'État déclarait aux universités qu'il n'y aurait pas d'investissement majeur sur le plan du soutien à l'équipement des autres facultés dans la mesure où ces dernières disposaient d'autres ressources. Ce faisant, on renvoyait la responsabilité de la construction d'une infrastructure informatique à la capacité d'investissement des institutions ou des professeurs-chercheurs.

Trois années ont passé depuis ce nouveau "virage technologique». Par delà les discours "technophiles ou technophobes», qu'en est-il de l'intégration des TIC en pédagogie universitaire en général et en formation à la profession enseignante en particulier? Pour tenter de répondre à cette question, nous devrons faire quelques détours en tentant d'établir une relation entre diverses variables qui déterminent ou qui affectent le rapport entre l'individu et les «nouvelles» technologies, notamment dans le cadre des programmes de formation de premier cycle.

Dans un premier temps, nous explorerons relativement rapidement la littérature scientifique internationale en ce qu'elle traite de façon générale des enjeux épistémologiques de l'intégration des TIC à la formation initiale en milieu universitaire et de façon plus spécifique du rôle des ces technologies en formation à la profession enseignante. Ce faisant, nous tenterons de «prendre la mesure» de la distance qui sépare un discours portant sur les bienfaits de l'intégration des TIC par rapport à l'état de la recherche scientifique sur les effets réels de cette intégration sur la relation enseignement-apprentissage.

Dans un deuxième temps, nous présenterons les résultats d'une enquête par questionnaire réalisée au printemps 1998 auprès d'un échantillon représentatif des enseignantes et des enseignants intervenant en formation initiale dans le cadre des divers programmes offerts à l'Université de Sherbrooke. Nous distinguerons, tour à tour, les compétences des intervenantes et des intervenants en matière d'utilisation de l'informatique et de la télématique, les profils de pratique d'utilisation de ces technologies à des fins didactiques et leurs attitudes à leur égard. Nous conclurons notre brève étude par une série de questions portant sur les enjeux épistémologiques et les choix curriculaires que soulève l'intégration des TIC dans les programmes de premier cycle universitaire. 


\section{L'intégration des TIC en pédagogie universitaire et leur insertion à la formation à la profession enseignante: deux réalités distinctes?}

Depuis la seconde moitié des années 1990, et tout particulièrement depuis l'explosion de l'accessibilité au réseau W3, on constate un développement rapide de la littérature scientifique traitant de l'intégration des TIC au sein des programmes de formation initiale. Bien qu'elle soit particulièrement "dynamique» dans l'univers anglo-saxon, cette littérature est en émergence dans l'ensemble des pays industrialisés et plus particulièrement au sein de la francophonie (Rhéaume et Laferrière, 1998). On peut la catégoriser en deux domaines généraux. D’une part, les écrits foisonnent en matière d'intégration des TIC en pédagogie universitaire, essentiellement dans l'univers anglo-américain ainsi qu'en Australasie ${ }^{1}$. D'autre part, toujours dans l'univers anglo-saxon, mais cette fois plutôt en Europe et aux États-Unis, on trouve une abondante littérature traitant de l'intégration de l'informatique et de la télématique en formation à la profession enseignante. $\mathrm{Si}$, dans le premier cas, on aborde la question ouvertement sous l'angle d'une réforme des fondements épistémologiques de la formation universitaire, cet angle est nettement moins présent lorsqu'on traite de formation à la profession enseignante.

\section{Le caractère épistémologiquement déterminé des TIC en tant que matériel didactique}

Depuis quelques années, nombre d'écrits portant sur l'intégration des TIC en tant qu'infrastructure didactique en pédagogie universitaire tendent à établir une relation plus ou moins explicite entre le recours aux environnements interactifs et une position épistémologique de type constructiviste (Dalgarno, 1996; Lefoe, 1998). Ces travaux impliquent deux postulats relativement complémentaires.

Le premier postulat sous-entend la présomption que les professeurs partagent une représentation de type constructiviste ou socioconstructiviste de la relation enseignement-apprentissage. Ce faisant, l'adoption des «nouvelles technologies», en ce qu'elles permettent une responsabilisation de l'apprenant ainsi qu'éventuellement l'interaction entre les apprenants, d'une part, et entre les apprenants et l'enseignant, d'autre part, serait le fruit d'une rupture profonde de ce dernier avec les approches traditionnelles ou béhavioristes de l'enseignement (Jonassen, Davidson, Collins, Campbell et Haag, 1995; Jonassen et Reeves, 1996; Lefoe, 1998).

Le second postulat implique la primauté du matériel didactique sur les orientations épistémologiques. Puisque le matériel informatique auquel on se réfère permet ou implique l'interaction, l'apprentissage en sera le produit. Le matériel didactique garantit donc la nature constructiviste de l'apprentissage et la dimension collabora-

1. Nous utilisons le «vocable» Australasie pour désigner les pays «industrialisés» du sud-est asiatique et de l'aire australe. Le terme est d'usage courant chez les Australiens afin de désigner de façon plus spécifique une communauté formée essentiellement de l'Australie, de la Nouvelle-Zélande, de Singapour et de la Malaisie. 
tive de l'enseignement (Choi et Yeom, 1996; Marton, 1994). Il est intéressant, par ailleurs, de constater qu'il s'agit là d'une représentation du rôle du matériel didactique fort semblable à celle que véhicule le ministère de l'Éducation du Québec depuis près de vingt ans maintenant (Gouvernement du Québec, 1979).

\section{Le profil d'utilisation des IIC en pédagogie universitaire: une fonction de l'orientation épistémologique de l'enseignant?}

Plusieurs auteurs suggèrent que sans certaines caractéristiques individuelles, tant sur le plan des "qualités traditionnelles" nécessaires à l'intervention pédagogique adéquate que sur celui de la maîtrise des TIC, l'insertion de ces dernières dans la pratique des professeurs peut s'avérer plus néfaste que bénéfique (De Kerckhove, 1997; Rhéaume, 1997). En réalité, la littérature scientifique en matière d'intégration des TIC en pédagogie universitaire se scinde en deux courants majeurs. Un premier courant, très puissant dans les pays «anglophones» du Pacifique et tout particulièrement en Australie, identifie l'intégration des TIC à la fois en tant que condition de survie des institutions universitaires et comme contexte favorisant la modification des pratiques d'enseignement dans une perspective épistémologique socioconstructiviste. Ce faisant, ces approches privilégient le développement de pratiques pédagogiques de type coopératif mettant en valeur l'intégration de logiciels environnements réseautés (Dalgarno, 1996; McNaught, 1996). Du côté francophone, ce courant trouve un écho particulièrement marqué chez les chercheurs et les praticiens qui œuvrent systématiquement dans le domaine de la formation à distance (Dufresne et Tremblay, 1991; Paquette, Paquin, Aubin et Ricciardi-Rigault, 1996).

Un second courant, plus pragmatique, considère que l'intégration des TIC en pédagogie universitaire n'est pas et ne sera pas particulièrement associée à la modification des pratiques pédagogiques et n'affectera pas en soi la position épistémologique des praticiens. L'enseignement demeurera essentiellement traditionnel, marqué par une perspective épistémologique de type néobéhaviorale. Les technologies de l'information remplacent simplement, en tant que matériel didactique, l'imprimé ou l'usage du tableau, de la craie ou du rétroprojecteur à faible résolution (Gosper et al., 1996; Tapper, 1997).

Ainsi, avec un peu de préparation et de soutien technique, mais sans faire varier la stratégie d'intervention éducative par rapport à l'approche universitaire traditionnelle, les pédagogues intègrent la saisie électronique de leurs notes de cours ou le manuel électronique plus ou moins interactif. Dans cette même perspective, ils peuvent prendre en considération la dimension «apprentissage» de la relation pédagogique et mettre à la disposition des étudiants divers outils dont ils gèreront seuls l'utilisation dans le cadre de leur cursus universitaire (Mcintyre et Wolff, 1998). C'est à ce type de préoccupation que répondent généralement l'intégration à l'arsenal universitaire d'outils de type logiciel d'autoévaluation formative "en ligne» ou encore l'accessibilité à des glossaires et à des compléments de notes de cours saisis en version hypertextuelle téléaccessible (Dirand et Larose, 1997; Larose, 1997, 1998; Nault, 1998).

Le postulat est identique, ici, à celui que Bloom formulait lors de l'élaboration du concept de pédagogie de la maîtrise (Bloom, 1979; Bloom et Broder, 1950). 
L'augmentation du temps et de la fréquence d'accès à l'information ainsi que la régularité des rétroactions, surtout si elles sont déchargées du poids affectif associé à l'évaluation sommative, garantissent l'apprentissage chez l'apprenant. Si ce dernier ne performe pas malgré tout, cela relèvera de ses caractéristiques individuelles, donc l'échec sera sa responsabilité et non celle de l'enseignant, du contexte d'enseignement ou de l'institution. Le recours à l'informatique ne met en cause ni les stratégies d'intervention éducative ni le rapport au savoir ainsi qu'à sa construction qu'entretient l'enseignant ${ }^{2}$. Le professeur est simplement mieux outillé (du moins en théorie) pour reproduire un modèle d'enseignement dans un contexte où il ne peut plus avoir de contacts individualisés avec l'apprenant, et ce dernier est mieux outillé pour compenser cette perte de contact individuel «en temps réel»ou, si l'on préfère, en situation de classe.

Si une quantité sans cesse croissante d'auteurs semble établir un parallèle entre l'intégration des TIC à l'enseignement et l'adoption d'une perspective constructiviste, l'accent mis sur la primauté de l'option épistémologique par rapport à la dimension technique demeure «faible». En fait, dans plusieurs cas cette prise de position semble plutôt formelle, la dimension centrale demeurant l'importance accordée à la qualité "technologique» du matériel didactique. Ainsi, Boulet (1998) accorde un paragraphe à la présentation de l'importance d'adopter une perspective constructiviste si les enseignantes et les enseignants désirent que l'intégration des TIC en classe favorise réellement l'apprentissage. Citant Jonassen et Reeves (1996), il présente les technologies multimédias en tant qu'outils cognitifs qui rendent possible en soi le développement de l'autonomie et de la coconstruction des savoirs:

[..] les outils cognitifs sont plus efficaces lorsqu'ils sont appliqués dans un environnement d'apprentissage constructiviste; les outils cognitifs permettent aux apprenants d'élaborer leur propre représentation de la connaissance plutôt que de les inciter à absorber des représentations préconçues par d'autres; les outils cognitifs peuvent favoriser le développement et l'utilisation d'une pensée réflexive profonde qui est nécessaire pour un apprentissage signifiant; les outils cognitifs permettent des apprentissages intéressants et exigeants pour l'esprit plutôt que les apprentissages faciles tels que promis, mais rarement réalisés par d'autres technologies éducatives; les outils cognitifs devraient être appliqués à des tâches ou à des problèmes définis par les apprenants avec l'appui de leurs enseignants; les outils cognitifs utilisés en éducation devraient être utilisés dans des contextes réalistes et permettre des résultats personnellement significatifs pour les apprenants; et, finalement, les outils cognitifs peuvent permettre un partenariat intellectuel sous la forme d'un traitement cognitif distribué (Boulet, 1998, p. 2).

2. L'objet principal de cet article n'étant pas de présenter nos positions au regard de la modélisation de I'intervention éducative et de ses fondements épistémologiques, le lecteur pourra se référer à: Larose et Lenoir (1998), Larose, Lenoir, Bacon et Ponton (1994) et Lenoir (1993, 1997). 
Certes, ce type de réflexion renvoie à une dimension d'interactivité qui peut s'avérer une condition préalable à la réalisation d'apprentissages dans une perspective socioconstructiviste. Cependant, le discours sur l'interactivité porte le plus souvent sur les conditions nécessaires au soutien de la motivation d'un apprenant qui se trouve souvent seul devant un univers relativement «éclaté». À preuve, la popularité actuelle de l'étude de Boshier, Mohapi, Moulton, Qayyum, Sadownik et Wilson (1997) dans laquelle les auteurs, après avoir analysé de façon systématique 127 sites de soutien ou d'accès à autant de cours universitaires, mettent l'accent sur la dimension «démotivante» de la structure et des contenus d'une bonne partie de ces "cours informatisés». Si une partie des cours ne font que reproduire sur le Web une conception «traditionnelle» du rapport au savoir (fonction de transmission d'une "vérité» de celui qui sait vers l'apprenant), à l'autre extrême l'aspect technologique, accrocheur, de l'environnement d'apprentissage est tellement important que l'apprenant risque fort de «s'y noyer». Dans les deux cas, l'aspect principal de la critique des auteurs porte sur l'impact de la qualité du matériel ou de l'environnement didactique sur la motivation de l'apprenant.

\section{Technologies et pédagogie universitaire}

Face à un discours «technophile» portant sur les vertus de l'informatique et des TIC en tant qu'outils didactiques, voire en tant que tuteurs, notamment en pédagogie universitaire, l'exploration de la littérature scientifique récente ne permet d'identifier que quelques recherches systématiques, fondées sur des collectes de données substantielles, tentant d'objectiver les avantages et inconvénients de cette intégration sur le plan pédagogique. Ces recherches peuvent être classées en trois catégories dont l'importance relative est fort variable. La première touche l'efficacité du recours aux TIC sous diverses formes en tant que moyen de favoriser ou de soutenir l'apprentissage. La seconde catégorie porte essentiellement sur l'analyse des attitudes des professeurs ou des étudiants face aux environnements informatiques. Enfin, la troisième concerne l'identification du degré de pénétration du recours aux TIC en pédagogie universitaire.

\section{L'efficacité des TIC en tant que soutien à l'apprentissage}

Il existe une tradition dans la littérature portant sur les «technologies de l'éducation", voulant que l'intégration des TIC favorise la réalisation d'apprentissages chez les étudiants universitaires (Boshier et al., 1997; Hosie, 1993; Laferrière et Rhéaume, 1998; Reeves, 1992; Wills et McNaught, 1996). Cependant, c'est au primaire et au secondaire que ce discours est à la fois le plus dense et le plus systématiquement fondé sur des recherches méthodologiquement rigoureuses. Dans une revue critique de la littérature portant sur les applications pédagogiques de l'ordinateur (APO) puis sur l'intégration des TIC en enseignement, Grégoire, Laferrière et Bracewell (1996) font état de nombreuses recherches démontrant l'efficacité du recours, dans certaines conditions, aux TIC en tant qu'environnement d'apprentissage ou encore en tant que matériel didactique. Cependant, la majeure partie des recherches auxquelles se réfèrent les auteurs concernent ce qu'il est convenu 
d'appeler les applications pédagogiques de l'ordinateur et, plus particulièrement, l'efficacité du recours aux environnements informatiques afin de permettre une plus grande individualisation de l'enseignement.

Les recherches plus récentes, qui portent généralement sur l'intégration des environnements multimédias et tout particulièrement des «technologies de réseaux» aux divers ordres d'enseignement, mettent généralement l'accent sur les retombées positives de ces initiatives sur la motivation des apprenants. Il n'y a pas, ou presque, d'études portant sur des clientèles en âge de fréquentation scolaire primaire ou secondaire, qui démontrent un lien de causalité, directe ou non, entre l'intégration des TIC et l'amélioration de l'apprentissage, du rendement scolaire ou de l'intégration des compétences présumées développées de la part des apprenants.

Par ailleurs, il n'existe que peu d'études portant sur l'évaluation des impacts réels de l'intégration des technologies de l'information et des réseaux sur l'apprentissage dans le contexte spécifique de l'enseignement supérieur. D'une part, cette insertion plus ou moins systématique demeure relativement récente. D’autre part, dès que nous posons la question de l'évaluation de l'impact différencié des TIC sur l'apprentissage, nous soulevons de façon concomitante l'enjeu des fondements épistémologiques de l'enseignement.

Si le recours aux TIC va de pair avec l'adoption d'une perspective constructiviste ou socioconstructiviste de la relation enseignement-apprentissage, les critères, les fondements et les objets d'évaluation doivent être conceptualisés de façon distincte par rapport à la perspective "traditionnelle», béhavioriste, de l'apprentissage. Dès lors, ce qui est remis en question c'est la perspective même de ce qui fonde l'apprentissage, perçu en tant que processus individuel, spatio-temporellement déterminé, et les indicateurs de ce processus sont multiples. L'évaluation renvoie alors à l'objectivation de mécanismes conceptuels complexes qui font l'objet de champs d'application pluriels (Dixon-Krauss, 1996). En bref, la question qui est alors ouverte est celle de la pertinence d'approches «novatrices» en matière de mesure et d'évaluation des apprentissages, telles que l'évaluation authentique ou l'évaluation par portfolio (Bacon et Bloom, 1995). Comme les mécanismes d'opérationnalisation de ces démarches évaluatives sont encore expérimentaux, la probabilité que celles-ci pénètrent rapidement et de façon systématique le milieu de la pratique enseignante est faible.

\section{Les attitudes au regard de l'informatique}

La majeure partie des recherches effectuées portent sur les attitudes des enseignants ou sur celles des étudiants au regard de l'informatique pris au sens large et, plus particulièrement, au regard des interactions entre l'individu et l'ordinateur. La majorité d'entre elles portent sur les construits d'anxiété et de stress en situation d'interaction avec l'ordinateur (Brown, 1996; Hudiburg, Ahrens et Jones, 1994; Hudiburg, Brown et Jones, 1993; McBride et Nagle, 1996; Ngin et Simms, 1996). Ce corpus de recherche découle d'un courant relativement récent, surtout présent aux États-Unis et à moindre raison en Grande-Bretagne ainsi qu'en Allemagne. Il s'agit d'un courant relativement identifié à la psychologie sociale ainsi qu'à la psychologie 
du travail, dont l'objet d'étude principal s'avère être les impacts de modifications apportées aux environnements de travail, notamment lors de changements technologiques majeurs.

Bien que les recherches soient relativement peu nombreuses sur les dimensions propres aux attitudes des apprenants ou des enseignants au regard de l'intégration des TIC, il s'agit bel et bien là d'un univers d'investigation en pleine croissance (Brock et Sulsky, 1994; Larose et al., 1998; Larose, Lafrance, Grenon, Roy et Lenoir, 1998; McBride et Nagle, 1996). Le construit d'anxiété par rapport aux environnements informatiques étant relativement nouveau, c'est dans le domaine du développement d'une instrumentation de mesure et d'évaluation valide et fidèle que se font les principaux développements (Chappell et Taylor, 1997; Potosky et Bobko, 1998; Yaghi, 1997). Malheureusement, lorsqu'elles traitent de l'utilisation de l'informatique en contexte scolaire, la majorité de ces recherches ne se situent pas dans le cadre d'une réflexion plus large portant sur les implications du concept d'anxiété par rapport à l'informatique sur la situation didactique ou sur les probabilités d'apprentissage des sujets.

\section{La pénétration du recours aux TIC en pédagogie universitaire et les causes des} limites observées

Comme nous l'avons mentionné précédemment, actuellement très peu d'études présentent des données stables au regard des profils d'utilisation des TIC chez les professeurs d'université. Lorsque de telles données existent, comme c'est le cas dans quelques études canadiennes récentes (Proulx et Campbell, 1997) ainsi que dans certaines recherches américaines plus anciennes (Geoghegan, 1994; Faseyitan et Hirschbuhl, 1992; Mackowiak, 1991), elles ne tiennent pas compte du type d'intégration des TIC à l'enseignement. Ce faisant, elles restreignent ou elles excluent toute réflexion sur la nature du lien qui devrait exister entre la représentation que les enseignants universitaires se font de la relation enseignement-apprentissage et le type de support technologique à l'enseignement auquel ils recourent .

\section{Technologies et formation à la profession enseignante}

Parallèlement à la littérature mentionnée ci-dessus, il existe un second corpus documentaire, centré celui-là sur l'intégration spécifique des TIC en formation initiale à l'enseignement. Cette littérature se situe dans une perspective de continuité par rapport à une tradition de recherche portant sur la faible transposition observée dans la pratique des jeunes enseignantes et enseignants sur le plan des «compétences informatiques» acquises durant la formation universitaire (Brummelhus et Plomp, 1994; Byard, 1995; Kay et Mellar, 1994). L'ensemble de ces recherches mène à un même constat. Peu importe la qualité des équipements informatiques mis à la disposition des praticiennes et des praticiens dans l'environnement scolaire et indépendamment de la quantité de cours à laquelle ceux-ci sont exposés durant leur formation initiale, le niveau de transfert des compétences acquises ou des apprentissages réalisés sur le plan de la pratique s'avère très faible. 
Que le discours et la formation portent sur ce qu'il était convenu d'appeler antérieurement les «applications pédagogiques de l'ordinateur» ou sur le produit plus actuel de l'intégration des technologies de type multimédia, les conclusions sont identiques. L'impact majeur de la formation sur les formés demeure sur le plan de l'utilisation "privée» de ces technologies et non sur celui de l'intégration de ces dernières aux stratégies d'intervention éducative qu'ils développeront au quotidien.

Ainsi, Burbules et Callister (1997) et Callister et Burbules (1998) reconnaissent une efficacité minimale, sur le plan de l'apprentissage, aux stratégies de formation et de sensibilisation à l'utilisation de l'informatique chez les professionnels de l'enseignement. Les auteurs soulignent que plusieurs d'entre eux, peu importe l'ordre d'enseignement, possèdent des compétences informatiques minimales, mais ne les utilisent pas sur le plan pédagogique dans la mesure où la rapidité d'obsolescence du matériel ainsi que des logiciels leur fait craindre une complexification indue et interminable de leur tâche. D'autres auteurs expliquent cette tendance par le faible niveau d'alphabétisation informatique ${ }^{3}$ qui caractérise les étudiantes et les étudiants au moment de leur intégration aux programmes de formation initiale à l'enseignement ainsi qu'au phénomène équivalent chez les jeunes professionnels de l'enseignement en exercice (Ferren, 1993; Lee, Pliskin et Kahn, 1994; Tapper, 1997).

\section{Le concept d'alphabétisation informatique}

Pour Mcmillan (1996), le concept d'alphabétisation informatique correspond à un univers théorique relativement flou et fluctuant, la définition du concept étant plus ou moins précise selon qu'elle se situe au niveau de l'identification d'habiletés «opérationnelles» ou à celui du discours sociopolitique. Comme la plupart des auteurs contemporains, il tend à centrer la définition de l'alphabétisation informatique autour d'un nombre restreint de compétences ou d'habiletés, identifiables en tant que comportements «ouverts» (Bradley, 1996; Reid, 1997; Stein, Craig et Scollary, 1997). Ces caractéristiques sont les suivantes:

- L'individu sait comment utiliser un logiciel de traitement de texte, un logiciel de courrier électronique et un fureteur (browser) lorsqu'il navigue sur l'Internet.

- Il est capable d'enregistrer ou de télécharger des informations sur une disquette (ou quelque autre unité de sauvegarde externe) afin de récupérer et d'imprimer ces dernières ailleurs.

À ces «compétences» comportementales, Stein, Craig et Scollary (1997) ajoutent une dimension cognitive, dimension qui est par ailleurs reconnue par la commission nationale de l'emploi, de l'éducation et de la formation d'Australie (NBEET, 1995). Il s'agit de l'habileté à utiliser les technologies de l'information pour identifier et retrouver de façon efficace des informations spécifiques dans le but de bâtir ou de s'approprier de la connaissance et de développer une pensée critique et créative.

3. Alphabétisation informatique: il s'agit ici de notre traduction du concept de « computer literacy». 


\section{Les variables qui affectent le degré d'alphabétisation informatique}

Le niveau d'alphabétisation informatique atteint par un individu peut être considéré de façon relative. Par exemple, dans les programmes des facultés d'éducation comme dans la plupart des programmes de formation initiale universitaires, les étudiantes et les étudiants tendent rapidement à s'équiper sur le plan informatique. Une proportion non négligeable des étudiantes et des étudiants possède donc un ordinateur et, généralement, maîtrise des habiletés minimales d'utilisation de logiciels de bureautique. Il s'agit d'ailleurs le plus souvent d'habiletés liées à l'utilisation de traitements de texte. Néanmoins, la proportion de celles et de ceux qui possèdent de façon concomitante un modem et qui considèrent avoir les moyens financiers de se payer un abonnement à un serveur commercial est nettement moindre.

Au moment de commencer des études universitaires, les étudiantes et les étudiants ont donc un degré variable d'alphabétisation informatique (Larose, 1998; Larose, Lafrance, Grenon, Roy et Lenoir, 1998a, 1998b). Ce niveau d'alphabétisation dépend essentiellement des expériences antécédentes d'accès à un ordinateur et d'utilisation libre ou ludique de ses diverses fonctions (Dugdale, Dekoven et Ju, 1998). À leur tour, ces expériences sont fonction de l'origine ethnoculturelle et de la classe sociale d'origine des individus (Hawkins et Paris, 1997).

\section{L'intégration des TIC en pédagogie universitaire ainsi qu'en formation initale à l'enseignement: portrait d'une université québécoise}

Durant le semestre d'hiver 1998 nous avons procédé à une enquête ${ }^{4}$ systématique auprès des membres du corps enseignant de l'Université de Sherbrooke afin d'identifier le niveau d'alphabétisation informatique du personnel, ses attitudes au regard des TIC et ses pratiques d'intégration des outils électroniques de soutien à l'enseignement. De mai à septembre 1998, nous avons procédé à deux collectes d'information successives. Dans un premier temps, nous avons réalisé une enquête au moyen d'un questionnaire diffusé auprès de l'ensemble des professeurs et d'un échantillon large de chargés de cours, pour autant qu'ils accomplissent au moins une partie de leur tâche au premier cycle. Dans un deuxième temps, nous avons réalisé cinquante-deux entrevues semi-structurées auprès d'autant d'enseignants, professeurs ou chargés de cours, ayant fait mention de leur volonté de participation lors de leur réponse au questionnaire d'enquête. Nous ne présenterons ici que certains résultats de l'enquête par questionnaire.

4. La recherche intitulée "Identification des attitudes et des pratiques d'utilisation des TIC en pédagogie universitaire» fait l'objet d'un soutien financier de la part du vice-rectorat à la recherche de l'Université de Sherbrooke (Fonds FRAl, volet Expérimentation en technologies de la formation). 


\section{Méthode}

Du 25 mai au 30 juin 1998, 800 questionnaires ont été expédiés à l'ensemble du corps professoral $(\mathrm{N}=600)$ ainsi qu'à un sous échantillon de 250 chargés de cours. Les questionnaires ont été acheminés aux sujets par voie de courrier intérieur lorsque ceux-ci disposaient de pigeonniers ou par courrier régulier lorsqu'ils ne disposaient pas d'un point fixe, attitré, de levée de courrier à l'intérieur de l'institution. Le mode d'échantillonnage sélectionné est simple. D'une part, le questionnaire a été expédié à tous les professeurs de l'institution sur la base de la liste des membres du corps professoral communiquée par les décanats de l'ensemble des facultés au 15 mai 1998. D’autre part, nous avons sélectionné aléatoirement $30 \%$ des chargés de cours identifiés selon la même source.

\section{Instrument}

L'instrument d'enquête utilisé est un questionnaire à structure de réponses fermées divisé en trois parties principales, outre la rubrique d'identification des sujets. Les questions de la première partie portent sur le type d'équipement détenu par les répondantes et les répondants, sur leur logithèque de prédilection ainsi que sur leur profil de compétence au regard de l'utilisation de divers environnements informatiques et télématiques. La seconde partie comporte des items centrés sur leurs pratiques d'utilisation de ces outils, tant sur le plan privé que sur celui de leur pratique pédagogique. Enfin, la troisième partie comporte dix-sept items ou énoncés à choix de réponse dichotomique formant une échelle d'attitude au regard de l'informatique.

\section{Échantillon}

Notre échantillon en est un de type «échantillon de convenance». Il est composé de 269 intervenants, travaillant en formation initiale au sein de cinquante départements répartis entre les neuf facultés de l'Université de Sherbrooke. Il s'agit d'un échantillon essentiellement masculin avec 76,6\% d'hommes et $23,4 \%$ de femmes. La majorité des répondants sont des professeurs répartis entre tous les échelons de la hiérarchie professionnelle. Les membres du corps professoral représentent $78,8 \%$ de l'échantillon, alors que la représentation des chargés de cours demeure minoritaire $(21,2 \%)$. Les variables sexe et statut professionnel sont significativement associées $\left(\mathrm{L}^{2}=31,68(4), \mathrm{p}<0,0001 \text {; V de Cramer }=0,334, \mathrm{p}<0,0001\right)^{5}$. La plus forte représentation de femmes se trouve au sein des deux catégories à la fois les plus instables sur le plan de l'emploi et dont l'influence sur la structure curriculaire est la plus faible, soit les chargés d'enseignement (42,9\% de la catégorie) et les chargés de cours ( $42,1 \%$ de la catégorie).

L'âge moyen des répondants de l'échantillon reflète un corps enseignant relativement jeune ( $\mu € 44,91 ; \sigma \in=8,70$ ); seuls les professeurs titulaires sont significativement plus âgés que leurs collègues occupant un autre rang professoral $(\mu € 51,77$;

5. $L^{2}$ : Likelihood ratio chi square ou chi carré probabiliste; $V$ de Caramer: mesure d'association permettant le croisement de variables à nombre de modalités inégal, indépendamment du format échantillonnal. 
$\sigma €=5,92)$. Les femmes de l'échantillon sont aussi significativement plus jeunes que les hommes ( $t=3,64$ (236), $\mathrm{p}<0,0001)$.

La majorité des répondants intervient de façon exclusive au niveau des programmes de formation initiale offerts dans les diverses facultés. La proportion de sujets intervenant de façon concomitante dans le cadre des programmes d'études supérieures demeure marginale. La majeure partie de l'échantillon est constituée d'enseignants chevronnés. Ils ont une expérience moyenne d'enseignement au premier cycle de 13,8 ans $(\sigma=9,08)$. Les professeurs titulaires se distinguent de l'ensemble de l'échantillon avec une expérience moyenne d'intervention au premier cycle de 22,39 ans.

\section{Résultats}

Une majorité d'enseignants de l'Université de Sherbrooke disposent d'un ordinateur, tant au bureau qu'au domicile. À l'université, 89,4\% des répondantes et des répondants déclarent avoir accès à un poste de travail individuel, alors que $92,4 \%$ d'entre eux possèdent au moins un ordinateur à la maison. Les répondantes et les répondants qui ne disposent pas d'un ordinateur appartiennent à deux catégories professionnelles particulières: les professeurs titulaires ainsi que les chargés de cours. Près des deux tiers de ceux qui possèdent un ordinateur à la maison peuvent entrer en relation avec le réseau universitaire par modem, alors que plus des trois quarts d'entre eux peuvent utiliser ce réseau depuis leur poste de travail au bureau. Les enseignants universitaires sont principalement des utilisateurs de logiciels de bureautique et, en tout premier lieu, de traitement de texte. S'ils disposent de logiciels environnements, par exemple Microsoft Office, ils n'en exploitent pas de façon égale les diverses fonctions. Ainsi, les logiciels de traitement de texte sont des outils auxquels on recourt beaucoup plus fréquemment que les tableurs (Excel) ou les logiciels de présentation (PowerPoint).

Si le recours aux logiciels de bureautique semble caractériser la majeure partie de l'échantillon, le niveau d'expertise que les sujets s'attribuent en la matière varie d'une faculté à l'autre. Ainsi, dans l'ensemble des facultés, la moitié ou plus des répondants s'autoévaluent en tant qu'utilisateurs moyens de ces logiciels. Cependant, en droit ainsi qu'en sciences et en sciences appliquées, près de la moitié des répondants considèrent en être des utilisateurs «experts».

La majeure partie du corps enseignant recourt aussi au moins marginalement au courrier électronique. Il n'y a qu'au sein des facultés d'éducation, d'éducation physique et sportive, de lettres et sciences humaines ainsi que de médecine qu'un pourcentage minimal des répondants n'utilisent jamais ce médium. De façon surprenante, c'est à la Faculté d'éducation qu'on trouve la plus forte proportion de nonusagers du courriel (12,1\% des répondants). De façon concomitante, c'est aussi à la Faculté d'éducation qu'on trouve le plus fort pourcentage de non-utilisateurs du réseau W3 (14,3\% des répondants). 
Une majorité de répondants n'utilisent pas, ou très marginalement, les moyens électroniques de soutien à l'enseignement (acétate électronique, canon à projection, vidéoconférence). Cependant, les deux premiers instruments sont d'usage plus fréquent - plus de $20 \%$ des répondants les utilisant parfois - que la conférence électronique, à laquelle $90 \%$ des sujets ne recourent jamais.

\section{L'utilisation de l'informatique en pédagogie universitaire}

Dans le questionnaire, quatre rubriques portaient sur l'identification de l'intégration de l'ordinateur, d'Internet, du courrier électronique ou encore d'environnements (logiciels) réseautés dans le cadre de la pratique d'enseignement des sujets. Dans un premier temps, nous constatons que le recours à l'ordinateur, les postes de travail étant utilisés de façon autonome ou réseautée par les étudiants, ne peut plus être considéré comme une réalité marginale à l'Université de Sherbrooke (tableaux 1 et 2). Par contre, le recours à la télématique dans le cadre de la didactique en formation initiale demeure une pratique relativement rare chez nos sujets.

Tableau 1. Pourcentage des fréquences d'utilisation des éléments de soutien informatique ou télématique

\begin{tabular}{|l|c|c|}
\cline { 2 - 3 } \multicolumn{1}{c|}{} & \multicolumn{2}{c|}{ Pourcentage } \\
\hline Instrument & N'utilise pas & Utilise \\
\hline Conférences électroniques (Courriel) & 86,3 & 13,7 \\
\hline Internet & 88,2 & 11,8 \\
\hline Ordinateurs & 51,4 & 48,6 \\
\hline $\begin{array}{l}\text { Ordinateurs reliés en réseau } \\
\text { (Environnements réseautés) }\end{array}$ & 58,8 & 41,2 \\
\hline
\end{tabular}

Tableau 2. Intégration de l'ordinateur à l'enseignement : pourcentage des répondants pondéré par faculté

\begin{tabular}{|l|c|c|}
\cline { 2 - 3 } \multicolumn{1}{c|}{} & \multicolumn{2}{c|}{ Pourcentage } \\
\hline Faculté & N'intègre pas & Intègre à son enseignement \\
\hline Administration & 28,0 & 72,0 \\
\hline Droit & 72,2 & 27,8 \\
\hline Éducation & 54,5 & 45,5 \\
\hline Éducation physique et sportive & 58,3 & 41,7 \\
\hline Lettres et sciences humaines & 50,0 & 50,0 \\
\hline Médecine & 58,3 & 41,7 \\
\hline Sciences & 57,1 & 42,9 \\
\hline Sciences appliquées & 24,0 & 76,0 \\
\hline Théologie, éthique et philosophie & 83,3 & 16,7 \\
\hline
\end{tabular}


Le calcul des mesures d'association entre l'appartenance facultaire et chacune des quatre variables décrivant le type de recours à l'informatique ou à la télématique dans l'enseignement ne permet d'identifier de relation significative qu'avec les variables «utilisation de l'ordinateur» d'une part ( $\mathrm{L}^{2}=21,46(8), p<0,006 ; \mathrm{V}=0,282$, $p<0,008)$ et «recours aux environnements réseautés» d'autre part $\left(\mathrm{L}^{2}=22,51(8)\right.$, $p<0,0008 ; \mathrm{V}=0,307, p<0,002$ ).

Un bref regard à la partition des fréquences d'utilisation de l'ordinateur réseauté, dans le cadre de l'enseignement de premier cycle, selon la faculté d'appartenance des sujets (tableau 3), permet de constater, là encore, une prédominance de l'intégration de ces technologies à la Faculté des sciences appliquées ainsi qu'à la Faculté d'administration. Inversement, c'est en théologie, éthique et philosophie ainsi qu'en droit que l'ordinateur (réseauté ou non) est le moins intégré aux pratiques d'enseignement en tant qu'outil didactique.

Tableau 3. Intégration d'environnements réseautés à l'enseignement : pourcentage des répondants pondéré par faculté

\begin{tabular}{|l|c|c|}
\cline { 2 - 3 } \multicolumn{1}{c|}{} & \multicolumn{2}{c|}{ Fréquence } \\
\hline Faculté & N'intègre pas & Intègre à son enseignement \\
\hline Administration & 28,0 & 72,0 \\
\hline Droit & 72,2 & 27,8 \\
\hline Éducation & 66,7 & 33,3 \\
\hline Éducation physique et sportive & 61,5 & 38,5 \\
\hline Lettres et sciences humaines & 61,4 & 38,6 \\
\hline Médecine & 68,9 & 31,1 \\
\hline Sciences & 55,9 & 44,1 \\
\hline Sciences appliquées & 36,0 & 64,0 \\
\hline Théologie, éthique et philosophie & 100,0 & 0,0 \\
\hline
\end{tabular}

La variable statut (rang professoral) s'avère significativement associée avec le fait d'utiliser ou non l'ordinateur en tant qu'outil didactique dans son enseignement $\left(\mathrm{L}^{2}=14,41(4), \mathrm{p}<0,006 ; \mathrm{V}=0,230, \mathrm{p}<0,008\right)$. Les enseignants à statut précaire intègrent l'informatique à leur pratique didactique de façon beaucoup moins systématique que ne le font leurs pairs ayant un statut permanent. Il suffit, pour s'en convaincre, de jeter un coup d'œil sur le tableau 4. Par ailleurs, si la variable sexe n'est pas directement associée aux pratiques d'intégration de l'ordinateur à la didactique au premier cycle, lorsqu' on l'utilise comme variable de contraste selon le statut professoral on constate qu'il y a un effet lorsqu'il s'agit des sujets masculins de l'échantillon ( $\mathrm{L} 2=13,70(4), \mathrm{p}<0,008 ; \mathrm{V}=0,256, \mathrm{p}<0,01$ ). 
Les technologies de l'information et de la communication en pédagogie universitaire et en formation à la profession enseignante: mythes et réalités

Tableau 4. Répartition de l'échantillon par statut professionnel selon le pourcentage des fréquences d'utilisation de l'ordinateur à des fins didactiques

\begin{tabular}{|l|c|c|}
\cline { 2 - 3 } \multicolumn{1}{c|}{} & \multicolumn{2}{c|}{ Pourcentage } \\
\hline Statut professionnel & N'intègre pas & Intègre à son enseignement \\
\hline Chargée ou chargé d'enseignement & 84,6 & 15,4 \\
\hline Professeure ou professeur adjoint & $\mathbf{5 6 , 5}$ & 43,5 \\
\hline Professeure ou professeur agrégé & 44,7 & $\mathbf{5 5 , 3}$ \\
\hline Professeure ou professeur titulaire & 42,9 & $\mathbf{5 7 , 1}$ \\
\hline Chargée ou chargé de cours & $\mathbf{6 4 , 3}$ & $\mathbf{3 5 , 7}$ \\
\hline
\end{tabular}

En bref, sur le plan de l'utilisation pédagogique des TIC, deux profils distincts apparaissent. Si plus de $40 \%$ des enseignants utilisent l'ordinateur, réseauté ou non, dans le cadre de leur enseignement, moins de $15 \%$ d'entre eux recourent à la télématique (courrier électronique et Internet) à des fins pédagogiques. L'utilisation de l'ordinateur à des fins pédagogiques est très significativement associée à certains profils facultaires. Ainsi, ce sont les enseignants des facultés d'administration et de sciences appliquées qui utilisent relativement systématiquement les ressources de l'ordinateur dans leur enseignement de premier cycle (plus de $70 \%$ des répondants de ces deux facultés), alors qu'en droit ainsi qu'en théologie, éthique et philosophie les proportions s'inversent. Dans les autres facultés, de $40 \%$ à $45 \%$ des répondants intègrent d'une façon ou d'une autre l'informatique à leur intervention didactique.

Attentes du corps enseignant au regard des compétences que les étudiantes et les étudiants devraient détenir par rapport aux TIC

Nous disposions de cinq items dont le but était d'identifier les compétences minimales qui devraient être détenues par les étudiantes et les étudiants dans les domaines suivants:

- utilisation d'un logiciel de traitement de texte;

- utilisation d'un tableur ou d'un chiffrier;

- utilisation du courrier électronique;

- navigation sur l'Internet;

- capacité de sauvegarder, de récupérer et d'imprimer de l'information sur une unité externe.

Chacune de ces «compétences» devait être cotée selon une «échelle» à quatre degrés allant de «sans importance» jusqu'à compétence «indispensable». Comme le lecteur pourra le constater (tableau 5), l'ensemble des compétences identifiées semble faire partie des éléments d'alphabétisation informatique qui devraient caractériser nos clientèles. 
Les technologies de l'information et de la communication en pédagogie universitaire et en formation à la profession enseignante: mythes et réalités

Tableau 5. Importance accordée à diverses compétences relevant de I'« alphabétisation informatique " que les étudiantes et les étudiants devraient détenir selon les répondantes et les répondants (pourcentage de l'échantillon)

\begin{tabular}{|l|c|c|c|c|}
\cline { 2 - 5 } \multicolumn{1}{c|}{} & \multicolumn{4}{c|}{ Importance } \\
\hline Compétence & Sans importance & Peu important & Très important & Indispensable \\
\hline Traitement de texte & 0,0 & 5,0 & 36,6 & 58,4 \\
\hline Tableur & 5,1 & 32,4 & 37,2 & 25,3 \\
\hline Courrier électronique & 1,9 & 17,2 & 39,1 & 41,8 \\
\hline Navigation sur I'Internet & 1,9 & 14,2 & 41,8 & 42,1 \\
\hline Sauvegarde et récupération & 0,8 & 4,2 & 38,9 & 56,1 \\
\hline
\end{tabular}

\section{Profil d'attitudes du corps enseignant}

Notre questionnaire comportait une liste de 17 items à réponse dichotomique portant sur trois domaines distincts et complémentaires. Une première série d'énoncés portait sur la perception des répondantes et des répondants au regard de l'utilité des TIC dans les dimensions privées de leur travail (préparation de cours, communication avec les collègues, recherches documentaires, etc.). Une seconde série portait sur la représentation que les sujets se font de l'utilité des TIC en tant qu'outils didactiques en enseignement au premier cycle. Enfin une troisième série d'énoncés portait sur le stress ressenti par les sujets lorsqu'ils sont confrontés à un environnement informatique.

Pour des motifs d'économie d'espace, nous ne présenterons pas ici les données de validation de notre instrument, mais ces dernières ainsi que ses propriétés métrologiques font l'objet de publications (Larose et al., 1998; Larose, David et Nault, à paraître). L'échelle générale ainsi que les trois sous-échelles constituées atteignent toutes un coefficient de consistance interne (alpha de Cronbach) satisfaisant, notamment lorsque nous tenons compte à la fois du format de l'échantillon ainsi que de l'univers de variation restreint qu'implique un format de réponse dichotomique (tableau 6).

Tableau 6. Coefficients de consistance interne (alpha de Cronbach) calculés pour l'échelle d'attitude générale ainsi que pour les sous-échelles

\begin{tabular}{|l|c|c|}
\hline Échelle & Alpha & N \\
\hline Échelle générale & 0,701 & 218 \\
\hline Attitude face à l'utilité de l'informatique sur le plan didactique & 0,664 & 235 \\
\hline Attitude face à l'utilité de I'informatique sur le plan individuel & 0,953 & 246 \\
\hline Anxiété (stress) par rapport aux environnements informatiques & 0,679 & 253 \\
\hline
\end{tabular}


À première vue, les enseignants ont une attitude générale plutôt positive à l'égard du recours à l'informatique (tableau 7). Cette attitude est cependant plus positive lorsqu'il s'agit du recours à l'informatique sur le plan individuel que sur celui de la pratique d'enseignement. Enfin, le niveau d'anxiété par rapport aux environnements informatiques demeure relativement peu élevé.

Tableau 7. Statistiques descriptives, scores calculés pour l'échelle d'attitude générale ainsi que pour les sous-échelles

\begin{tabular}{|l|c|c|c|c|c|}
\hline Variable & Moyenne & Écart-type & Score minimal & Score maximal & N \\
\hline Attitude générale & 22,52 & 2,55 & 14,00 & 26,00 & 218 \\
\hline $\begin{array}{l}\text { Utilité sur le plan } \\
\text { didactique }\end{array}$ & 6,95 & 0,45 & 5,00 & 8,00 & 246 \\
\hline $\begin{array}{l}\text { Utilité sur le plan } \\
\text { personnel }\end{array}$ & 9,83 & 1,66 & 6,00 & 12,00 & 235 \\
\hline $\begin{array}{l}\text { Anxiété relativement } \\
\text { à l'utilisation de } \\
\text { I'informatique }\end{array}$ & 4,11 & 1,13 & 3,00 & 6,00 & 253 \\
\hline
\end{tabular}

Les variables par rapport auxquelles les sujets se distinguent

Le calcul du $t$ de Student permet d'identifier une différence significative entre les moyennes observées selon le sexe par rapport à la variable «anxiété face aux environnements informatiques" $(t=-2,73(91,17) ; \mathrm{p}<0,008)$, les variances n'étant pas homogènes ( $\mathrm{F}$ de Levene $=15,57 ; p<0,0001$ ). Dans ce cas, le niveau d'anxiété enregistré est plus élevé chez les femmes $(\mu € 4,48 ; \sigma \in=1,28)$ que chez les hommes $(\mu €=3,98 ; \sigma \in 1,05)$.

L'analyse de variances unidimensionnelle permet d'identifier des différences significatives entre moyennes observées à la sous-échelle «anxiété par rapport aux environnements informatiques", selon le statut professionnel des répondants ( $F=2,94$ (4), $p<0,02$ ), les variances n'étant cependant pas jugées homogènes ( $F$ de Levene $=$ $5,27(4,248) ; \mathrm{p}<0,0001)$. Les professeurs adjoints affichent un niveau d'anxiété significativement plus élevé $(\mu € 4,63 ; \sigma €=1,27$ ) que leurs collègues titulaires ( $\mu €=3,86$; $\sigma \in=1,04$ ), le test de Bonferroni étant significatif au seuil $p<0,05$. On observe aussi des différences significatives entre les moyennes selon la faculté d'attache des répondantes et des répondants pour l'ensemble des échelles utilisées (tableau 8). 
Les technologies de l'information et de la communication en pédagogie universitaire et en formation à la profession enseignante: mythes et réalités

Tableau 8. Résultats des analyses de variance (unidimensionnelles) réalisées entre les variables métriques et la variable "faculté d'appartenance "

\begin{tabular}{|l|c|c|c|c|c|}
\hline Échelle & $\mathbf{F}$ & $\mathbf{d l}$ & $\mathbf{P}$ & $\begin{array}{c}\text { Homogénéité } \\
\text { des variances }\end{array}$ & Bonferroni \\
\hline Attitude générale & 22,52 & 2,55 & 14,00 & 26,00 & 218 \\
\hline $\begin{array}{l}\text { Utilité sur le plan } \\
\text { didactique }\end{array}$ & 6,95 & 0,45 & 5,00 & 8,00 & 246 \\
\hline $\begin{array}{l}\text { Utilité sur le plan } \\
\text { personnel }\end{array}$ & 9,83 & 1,66 & 6,00 & 12,00 & 253 \\
\hline $\begin{array}{l}\text { Anxiété relativement } \\
\text { à l'utilisation de } \\
\text { l'informatique }\end{array}$ & 4,11 & 1,13 & 3,00 & 6,00 & \\
\hline
\end{tabular}

En ce qui concerne l'échelle générale, on constate une différence significative entre le profil d'attitude des répondants de la Faculté d'éducation, d'une part, et ceux de la Faculté des sciences appliquées, d'autre part. Les gens de cette dernière faculté manifestent une attitude générale plus positive que leurs collègues d'éducation au regard des TIC. En ce qui concerne l'échelle d'attitude au regard de l'utilité didactique des TIC, les gens d'administration ont un profil d'attitude significativement plus favorable à cet effet que leurs collègues de droit et de la Faculté de théologie, d'éthique et de philosophie. En ce qui a trait à l'échelle d'attitude au regard de l'utilité personnelle des TIC, les gens d'administration affichent une attitude significativement plus positive que les répondantes et les répondants de droit et de la Faculté d'éducation. Enfin, les enseignants de sciences appliquées ont un niveau d'anxiété par rapport à l'informatique significativement plus faible que leurs collègues de la Faculté de théologie, d'éthique et de philosophie et de la Faculté d'éducation.

Selon qu'ils disposent ou non d'un ordinateur à la maison ou au bureau, les sujets ont des attitudes distinctes. Ainsi, les répondants qui disposent d'un ordinateur au bureau ont une attitude générale significativement plus favorable au regard des TIC que celles et ceux qui n'en disposent pas ( $t=2,43(207), p<0,02)$. Ces sujets affichent aussi un niveau d'anxiété beaucoup plus faible au regard des environnements informatiques que leurs collègues ( $t=3,69$ (242), $p<0,0001)$. Lorsqu'ils disposent d'un ordinateur à leur domicile, les sujets ont un score significativement plus élevé que leurs collègues à la sous-échelle «utilité personnelle» $(t=3,34(225), p<0,001)$ ainsi qu'à l'échelle d'attitude générale au regard des TIC (2,98 (208) $p<0,003)$.

Enfin, nous avons réalisé une série d'analyses multivariées de la variance (MANOVA) entre, d'une part, les variables métriques dont nous disposions (échelle et sous-échelles d'attitude) et, d'autre part, la faculté d'appartenance ainsi que le statut professionnel. L'interaction entre ces deux variables n'affectait que la distribution de la mesure de l'attitude au regard de l'utilité didactique de l'informatique en pédagogie universitaire ( $\mathrm{F}=2,28(227,10), p<0,015)$, les variances étant présumées homogènes (test de Cochran non significatif), l'interaction expliquant $18 \%$ de la variance totale. 


\section{Le recours à l'informatique à la Faculté d'éducation}

Sur le plan du degré d'alphabétisation informatique des professeurs, de leurs pratiques privées d'utilisation des TIC ainsi que de leurs attentes quant aux compétences informatiques que devraient détenir les étudiantes et les étudiants, les formatrices et les formateurs de la Faculté d'éducation ne se distinguent pas du profil général du corps professoral de l'Université. Par ailleurs, sur le plan de l'intégration des TIC à leurs propres pratiques d'enseignement, ces professeures et professeurs ont un profil semblable à celui des praticiennes et des praticiens des facultés de sciences humaines. Ils font partie de celles et de ceux qui recourent le moins fréquemment à ces moyens didactiques. Enfin, un élément distingue le sous-échantillon enseignant de la Faculté d'éducation de l'ensemble de l'échantillon. Les professeures et les professeurs de cette faculté ont une attitude nettement moins favorable que leurs collègues au regard de l'utilisation pédagogique des TIC $(t=2,74$ (215), $p<0,007$ ) et un sentiment d'anxiété significativement plus élevé que leurs consoeurs et confrères à cet égard $(t=2,13(250), p<0,03)$.

Pour terminer, nous avons tenté de voir si certaines variables n'affectaient pas spécifiquement ce profil d'intégration, tant à la Faculté d'éducation que dans l'ensemble des facultés. Nos résultats suggèrent que les enseignantes et les enseignants à statut précaire sont celles et ceux qui recourent le moins systématiquement aux TIC dans leur pratique didactique. À la Faculté d'éducation, ce sont essentiellement des professeurs titulaires qui utilisent les environnements informatiques, réseautés ou non, dans le cadre de leur enseignement. Dans l'ensemble des autres facultés, ce sont surtout des professeurs agrégés qui recourent à l'informatique en enseignement et ils tendent à intégrer plus systématiquement la télématique dans leurs cours.

\section{Discussion}

D’une manière générale, les données de notre recherche s'avèrent cohérentes avec l'état de la littérature scientifique portant sur les profils d'intégration des TIC en pédagogie universitaire. Le degré d'alphabétisation informatique du corps professoral s'avère satisfaisant, mais il n'y a que peu de transfert des compétences construites vers la pratique enseignante. La disparité entre facultés quant au rythme d'adoption des instruments didactiques à caractère informatique est aussi conforme à ce que suggère la littérature. Cette pénétration est supérieure dans les facultés de sciences appliquées et inférieure ou minimale dans les facultés de sciences humaines. Seul phénomène particulier, l'omniprésence du recours aux TIC ainsi qu'aux moyens électroniques de soutien à l'enseignement à la Faculté d'administration.

Cette donnée vient confirmer l'importance que la littérature scientifique accorde à la présence d'un soutien technique et logistique pour que le professorat intègre l'informatique à sa pratique professionnelle. La Faculté d'administration de l'Université de Sherbrooke a emménagé, il y a moins d'un an, dans de nouveaux locaux conçus en vue d'une utilisation optimale des TIC en enseignement. En outre, cette faculté s'est dotée de façon autonome d'un parc informatique impressionnant 
et de services techniques conséquents. Ces dimensions de soutien technique et de pertinence technologique font partie des critères que la CRÉPUQ (1997) énonçait en tant que conditions de base au recours systématique à l'informatique en pédagogie universitaire.

L'absence de différence de profil au regard des pratiques d'intégration pédagogique des TIC chez les professeures et les professeurs de la Faculté d'éducation, par rapport à leurs collègues de sciences humaines et de lettres, nous inquiète. Si nous tenons compte du fait que le ministère de l'Éducation exige que les curriculums de formation initiale à la profession enseignante incluent une dimension d'enseignement de l'utilisation pédagogique des TIC, le faible niveau d'intégration des TIC aux pratiques pédagogiques des professeures et des professeurs ne suggère qu'une conclusion. La sensibilisation à l'utilisation pédagogique des TIC se limite à l'intégration d'un ou deux cours spécifiques, thématiques, dans les programmes de formation. On maintient donc le statut de «matière scolaire» accordé à l'informatique pédagogique.

Il y a une distance impressionnante entre le fait d'utiliser un ordinateur en tant qu'objet d'apprentissage et celui de recourir à cet instrument en tant que support à l'apprentissage. La littérature scientifique en technologie éducative ne cesse d'affirmer que l'identification exclusive de l'informatique à un contexte "disciplinaire» représente une condition «adverse » par rapport à la probabilité que l'enseignante ou l'enseignant en formation effectue un transfert vers la pratique pédagogique des éventuelles compétences construites (Brummelhuis et Plomp, 1994). N'est-ce pas là l'exacte situation observée? Et, si tel est le cas, ne serait-il pas doublement important qu'un effort particulier soit consenti de la part du professorat de la Faculté d'éducation, dans le sens d'une intégration plus systématique des outils informatiques aux pratiques d'enseignement-apprentissage?

Pour notre part, nous croyons que cette intégration s'avère d'autant plus indispensable que la probabilité est forte que les étudiantes et les étudiants ne risquent guère d'être exposés à des pratiques systématiques d'utilisation pédagogique des TIC dans le cadre de leur formation pratique. À titre d'exemple, moins de $3 \%$ des enseignantes et des enseignants intervenant dans les écoles primaires des quatre commissions scolaires de la région de l'Estrie utilisent de façon plus ou moins régulière l'informatique ou la télématique dans leur pratique pédagogique. En conséquence, l'exposition à des pratiques d'enseignement utilisant les TIC de façon systématique dans le milieu universitaire représente probablement la seule possibilité que les apprenantes et les apprenants puissent identifier l'informatique en tant qu'instrument utile à la réussite sur le plan de la formation initiale. Si cette condition est remplie, alors les intervenantes et intervenants novices pourront effectuer un transfert des compétences acquises, jugées utiles, vers leur pratique professionnelle et, de cette façon, intègreront plus facilement le recours à l'environnement informatique dans leur propre enseignement. 


\section{Conclusion}

Au départ de cette enquête, les chercheurs désiraient simplement vérifier si le constat de Proulx et Campbell (1997) concernant l'inégalité de la pénétration des TIC en pédagogie universitaire, selon les facultés, s'appliquait aussi dans le contexte particulier d'une université québécoise. Outre la réponse positive à cette question, cette recherche nous a essentiellement permis de mettre en lumière la distance qui sépare le discours portant sur les bienfaits de l'intégration des technologies de l'information et de la communication par rapport à la réalité des pratiques enseignantes. Par ailleurs, il est intéressant de constater que ce discours, lorsqu'il tente de se fonder sur le plan théorique, renvoie à un univers conceptuel qui demeure tout aussi distant des pratiques observées sur le plan de la pédagogie universitaire, du moins en formation initiale. La justification actuelle du «virage technologique» se fonde sur un virage épistémologique de type constructiviste. Néanmoins, la structure même de la division en unités d'enseignement fermées qui caractérise les curriculums de premier cycle et la hiérarchisation ainsi que la présentation séquentielle des objectifs qui composent la majeure partie des plans de cours renvoient à une logique curriculaire de type bloomienne ou néobéhaviorale.

L'émergence d'un virage constructiviste, sinon socioconstructiviste, qui soustendrait la pénétration réelle des TIC au sein des pratiques enseignantes, impliquerait une modification profonde, sinon une remise en question globale, du rapport au savoir chez les membres du corps professoral. Cela impliquerait, à son tour, l'existence préalable d'un débat à caractère épistémologique portant sur les finalités des diverses formations initiales ainsi qu'une profonde remise en question du rôle même de la «personne enseignante». Un tel débat et une telle remise en question ne nous semblent guère marquer l'avant-scène du monde universitaire québécois.

Dans un tel contexte, quel avenir peut-on prévoir pour l'intégration des TIC dans nos programmes de premier cycle? La question reste ouverte. Sans remise en question globale des orientations épistémologiques qui fondent les rapports au savoir ainsi qu'aux processus d'acquisition de connaissances ou de construction de compétences, l'intégration systématique de la télématique et des technologies de réseaux à l'arsenal didactique en usage dans nos universités risque fort de se trouver cantonnée à l'univers des programmes de télé-enseignement. Peut-on dépasser l'état actuel des choses et comment peut-on y arriver? Là encore, la question reste ouverte.

\section{Références bibliographiques}

BACON, E. H. et BLOOM, L. A. (1995). Beyond the herring sandwich phenomenon A holistic constructivist approach to teacher education. Journal of Learning Disabilities, 28(10), 636-645. 
BLOOM, B. S. (1979). Caractéristiques individuelles et apprentissage scolaire. Bruxelles, Nathan/Labor.

BLOOM, B. S. et BRODER, L. (1950). Problem Solving Processes of College Students. Chicago, IL, University of Chicago Press.

BRADLEY, D. (1996). Integration of Information Techniques into Teaching and Learning. Canberra, University of South Australia.

BOSHIER, R., MOHAPI, M., MOULTON, G., QAYYUM, A., SADOWNIK, L. et WILSON, M. (1997). Best and worst dressed Web courses: Strutting into the $21^{\text {st }}$ century in comfort and style. Distance Education - An International Journal, 18(2), 36-49.

BROCK, D. B. et SULSKY, L. M. (1994). Attitudes toward computers: Construct validations and relations to computer use. Journal of Organizational Behavior, 15(1), 17-35.

BROWN, S. (1996). Organisational and cultural implications of changes in teaching and learning. Dans F. Brown (dir.), Proceedings of the ASCILITE96 Conference (p. 3-14). Adélaïde (Australie), Australasian Society for Computers in Terciary Education (ASCILITE).

BRUMMELHUS, A. T. et PLOMP, T. (1994). Computers in primary and secondary education: The interest of an individual teacher or a school policy? Computers Education, 22(4), 291-299.

BURBULES, N. C. et CALLISTER, T. A. (1997). Who lives here? Access to and credibility within cyberspace. Dans C. Bigum, et C. Lankshear (dir.), Digital Rhetorics: New Technologies, Literacy, and Learning. Current Practices and New Directions. Brisbane (Australie), Queensland University of Technology, p. 21-57.

BYARD, M. J. (1995). IT under school-based policies for initial teacher training. Journal of Computer Assisted Learning, 11(3), 128-140.

CALLISTER, T. A. et BURBULES, N. C. (1998). Paying the piper: The educational cost of the commercialization of the Internet. Electronic Journal of Sociology, 3(3). En ligne [http://www.sociology.org/vol003.003/index.html].

CHAPPELL, K. K. et TAYLOR, C. S. (1997). Evidence for the reliability and factorial validity of the computer game attitude scale. Journal of Educational Computing Research. 17(1), 67-77.

CHOI, Y. J. et YEOM, S. J. (1996). Virtual classes. Dans F. Brown (dir.), Proceedings of the ASCILITE96 Conference. Adélaïde (Australie), Australasian Society for Computers in Terciary Education (ASCILITE), p. 93-100.

DALGARNO, B. (1996). Constructivist computer-assisted learning: Theory and techniques. Dans F. Brown (dir.), Proceedings of the ASCILITE96 Conference. Adélaïde (Australie), Australasian Society for Computers in Terciary Education (ASCILITE), p. 127-148. 
DE KERCKHOVE, D. (1997). Connected Intelligence. Toronto, Somerville House Publishing.

DIRAND, J.-M. et LAROSE, F. (1997). Rapport sur le projet «Développement d'un système générique de didacticiels sur JAVA et $W W W »$. Sherbrooke, Université de Sherbrooke, Faculté des sciences appliquées, Département de génie électrique et informatique. Rapport de recherche présenté au vice-rectorat à l'enseignement.

DIXON-KRAUSS, L. (1996). Vygotsky in the Classroom: Mediated Literacy Instruction and Assessment. New York, Addison-Wesley Publ.

DUFRESNE, A. et TREMBLAY, I. (1991). Modelling distributed knowledge to support learning environments in physiology and computer science. International Conference on Simulation in Engineering Education. Simulation Series, 24(2), 185-189.

DUGDALE, S., DEKOVEN, E. et JU, M. K. (1998). Computer course enrollment, home computer access and gendet-relationships to high-school students success with computer spreadsheet use for problem solving in pre-algebra. Journal of Educational Computing Research, 18(1), 49-62.

FASEYITAN, S., LIBII, J. N. et HIRSCHBUHL, J. (1996). An inservice model for inhancing faculty computer self-efficacy. British Journal of Educational Technology, 27(3), 214-226.

FASEYITAN, S. et HIRSCHBUHL, J. (1992). Computers in university instruction: What are the significant variables that influence adoption? Interactive Learning International, 8(3), 185-194.

FERREN, A. S. (1993). General education reform and the computer revolution. The Journal of General Education, 42(3), 164-177.

GEOGHEGAN, W. H. (1994). What Ever Happened to Instructional Technology? Communication présentée à la $22^{\mathrm{e}}$ conférence annuelle de l'International Business Schools Computing Association, Baltimore, Maryland, 17-20 juillet. En ligne [http://ike.engr.washington.edu/news/whitep/whg/wpi.htm].

GOSPER, M., ANDREWS, J., HESKETH, B. et SABAZ, M. (1996). Electronic communication in university teaching: Expectations and implication over the next five years. Dans F. Brown (dir.), Proceedings of the ASCILITE96 Conference. Adélaïde (Australie), Australasian Society for Computers in Terciary Education (ASCILITE), p. 235-246.

GOUVERNEMENT DU QUÉBEC (1979). Énoncé de politique et plan d'action (Livre orange). Québec, Ministère de l'Éducation du Québec.

GOUVERNEMENT DU QUÉBEC (1996a). Conférence socio-économique sur les technologies de l'information et des communications en éducation au Québec: État de situation. Québec, Ministère de l'Éducation. [http://www.meq.gouv.qc.ca/m_pub.htm] 
GOUVERNEMENT DU QUÉBEC (1996b). Les technologies de l'information et de la communication en éducation. Plan d'intervention. Éducation préscolaire, enseignement primaire et secondaire, formation générale des jeunes et des adultes. Québec, Ministère de l'Éducation du Québec. En ligne [http://www.meq.gouv.qc.ca/m_pub.htm].

GRÉGOIRE, R., BRACEWELL, R. et LAFERRIÈRE, T. (1996). L'apport des Nouvelles Technologies de l'Information et de la Communication (NTIC) à l'apprentissage des élèves du primaire et du secondaire. Revue documentaire. Ottawa, Réseau scolaire canadien (RESCOL). En ligne [http://www.fse.ulaval.ca/fac/tact/fr/html/apport/apport96.html].

HAWKINS, R. et PARIS, A. E. (1997). Computer litteracy and computer use among college students. Differences in black and white. Journal of Negro Education, 66(2), 147-158.

HOSIE, P. (1993). Technologically mediated learning: The future of training in Australia. Australian Journal of Educational Technology, 9(1), 69-86.

HUDIBURG, R. A., AHRENS, P. K. et JONES, T. M. (1994). Psychology of computer use: XXXI. Relating computer users'stress, daily hassles, somatic complaints, and anxiety. Psychological Reports, 75(2), 1183-1186.

HUDIBURG, R. A., BROWN, S. R. et JONES, T. M. (1993). Psychology of computer use: XXIX. Measuring computer users'stress: The computer hassles scale. Psychological Reports, 753(1), 923-929.

JONASSEN, D., DAVIDSON, M., COLLINS, C., CAMPBELL, J. et HAAG, B. B. (1995). Constructivism and computer-mediated communication in distance education. The American Journal of Distance Education, 9(2), 7-26.

JONASSEN, D. H. et REEVES, T. C. (1996). Learning with technology: Using computers as cognitive tools. Dans D. H. Jonassen (dir.), Handbook of Research on Educational Communications and Technology. New York, Macmillan, p. 693-719.

KAY, J. J. et MELLAR, H. G. (1994). Information technology and new primary school teachers. Journal of Computer Assisted Learning, 10(2), 157-167.

LAROSE, F. (1997). Rapport d'activité partiel concernant l'implantation et l'utilisation des outils conventionnels d'autoévaluation informatisée ainsi que le recours au babillard électronique facultaire dans le cadre du cours PEP 122 offert aux étudiantes et aux étudiants de première année du baccalauréat en enseignement au préscolaire et au primaire. Sherbrooke, Université de Sherbrooke, Faculté d'éducation. Rapport de recherche présenté au Vicerectorat à l'enseignement. 
LAROSE, F. (1998). Le recours aux technologies de l'information et de la communication en pédagogie universitaire. Réflexion sur la réalité des étudiantes et des étudiants de première année en formation initiale à la profession enseignante au primaire. Sherbrooke, Université de Sherbrooke, Faculté d'éducation. Rapport de recherche présenté au Vice-rectorat à l'enseignement.

LAROSE, F., DAVID, R., DIRAND, J.-M., LENOIR, Y. et ROY, G.-R. (1998). Rapport de recherche portant sur le profil d'utilisation des TIC en pédagogie universitaire à Sherbrooke. Sherbrooke, Université de Sherbrooke, Vice-rectorat à l'enseignement.

LAROSE, F., DAVID, R. et NAULT, T. (à paraître). Une mesure adaptée des attitudes au regard de l'informatique chez les enseignants intervenant au premier cycle à l'Université de Sherbrooke. Mesure et évaluation en éducation.

LAROSE, F., LAFRANCE, S., GRENON, V., ROY, G.-R. et LENOIR, Y. (1998a). Du discours officiel à la prise en considération des attitudes des clientèles face à l'informatique en pédagogie universitaire. Bilan d'une enquête menée à la Faculté d'éducation de l'Université de Sherbrooke. Éducatechnologiques. En ligne [http://www.fse.ulaval.ca/fac/ten/reveduc/].

LAROSE, F., LAFRANCE, S., GRENON, V., ROY, G.-R. et LENOIR, Y. (1998b). Attitudes et compétences des étudiants en formation initiale à l'enseignement face à l'informatique à la Faculté d'éducation de l'Université de Sherbrooke. Brock Education (à paraître, printemps 1999).

LEE, D. M. S., PLISKIN, N. et KAHN, B. (1994). The relationship between performance in a computer literacy course and students' prior achievement and knowledge. Journal of Educational Computing Research, 10(1), 63-77.

LEFOE, G. (1998). Creating Constructivist Learning Environments on the Web: The Challenge in Higher Education. Wollongong (Aust.), $15^{\text {th }}$ Proceedings of Australasian Society for Computers in Tertiary Education (ASCILITE). En ligne [http://cedir.uow.edu.au/ASCILITE98/ascpapers98.html].

MACKOWIAK, K. (1991). The effects of faculty characteristics on computer applications in instruction. Journal of Research on Computing in Education, 23(3), 396-410.

MARTON, P. (1994). La conception pédagogique de Systèmes d'apprentissage multimédia interactif: fondements, méthodologie et problématique. Éducatechnologiques, 1(3). En ligne [http://www.fse.ulaval.ca/fac/ten/reveduc/html/voll/voll_no3.html].

MCBRIDE, S. H. et NAGLE, L. M. (1996). Attitudes toward computers. A test of construct validity. Computers and Nursing, 14(3), 164-170.

MCINTYRE, D. R. et WOLFF, F. G. (1998). An experiment with WWW interactive learning in university education. Computers and Education, 31(3), 255-264. 
MCMILLAN, S. (1996). Literacy and computer literacy. Definitions and comparisons. Computers and Education, 27(3-4), 161-170.

MCNAUGHT, C. (1996). The nature of information technology and its implications for practices and policy in higher education. Dans F. Brown (dir.), Proceedings of the ASCILITE96 Conference. Adélaïde (Australie), Australasian Society for Computers in Terciary Education (ASCILITE), p. 381-390.

NAULT, T. (1998). Enseigner et apprendre sur un site Web. Lyon, Document déposé dans le cadre du Symposium international franco-canadien «La formation des enseignants et des formateurs aux nouveaux usages et aux nouvelles pratiques des technologies de l'information et des réseaux». (ARDEMI, 3-4 décembre 1998).

NGIN, P. M. et SIMMS, L. M. (1996). Computer use for work accomplishment. A comparison between nurse managers and staff nurses. Journal of Nursing Administration, 26(3), 47-55.

NBEET (1995). Education and Technology Convergence: A survey of technology infrastructure in education and the professional development and support of educators and trainers in information and communication technologies. Canberra (Australie), National Board Employment Education and Training Commissioned Report No. 41.

PAQUETTE, G., PAQUIN, C., AUBIN, C. et RICCIARDI-RIGAULT, C. (1996). Définition du campus virtuel. Objectifs, méthodes, outils et principes de développement. Montréal, LICEF.

POTOSKY, D. et BOBKO, P. (1998). The computer understanding and experience scale. A self-report measure of computer experience. Computers in Human Behavior, 14(2), 337-348.

PROULX, M. et CAMPBELL, B. (1997). The professional practices of Faculty and the diffusion of computer technologies in university teaching. Electronic Journal of Sociology, 3(2). En ligne [http://www.sociology.org/vol002.003/proulx.article.1997.html].

REEVES, T. (1992). Research foundations for interactive multimedia. Dans Promaco (dir.), Proceedings of the International Interactive Multimedia Symposium. Applecross (Australie), Promaco Conventions Pty, p. 177-190.

REID. I. (1997). Computer Literacy in Higher Education. Perth (Australie), Paper presented to the 1997 ASCILITE Conference. En ligne [http://www.curtin.edu.au/conference/ASCILITE97/papers/Reid/Reid.html].

RHÉAUME, J. (1997). Enseigner aux grands groupes, par où commencer? La nouvelle rhétorique éducative. Québec, Université Laval, Faculté des sciences de l'éducation. Communication présentée dans le cadre du colloque CANTIC 97 (Colloque annuel sur les Nouvelles Technologies de l'Information et de la Communication). En ligne [http://www.fse.ulaval.ca/ jrheaume/oparleur.html\#resu]. 
RHÉAUME, J. et LAFERRIÈRE, T. (1998). Les technologies de l'information et de la communication à l'école: les futurs maîtres et les ressources disponibles. Lyon, Document déposé dans le cadre du Symposium international franco-canadien «La formation des enseignants et des formateurs aux nouveaux usages et aux nouvelles pratiques des technologies de l'information et des réseaux" (ARDEMI, 3-4 décembre 1998).

STEIN, A., CRAIG, A. et SCOLLARY, A. (1997). Preparatory IT practices and skills of transition business students. Australian Journal of Educational Technology, 13(1), 40-53.

TAPPER, J. (1997). Integrating online literacy into undergraduate education. Higher Education Research and Development, 16(1), 25-40.

WILLS, S. et C. MCNAUGHT (1996). Evaluation of computer-based learning in higher education. Journal of Computing in Higher Education, 7(2), 106-128.

YAGHI, H. M. (1997). Pre-university students attitudes toward computers. An international perspective. Journal of Educational Computing Research, 16(3), 237-249.

YETTON, P. (1997). Managing the Introduction of Technology in the Delivery and Administration of Higher Education. Canberra (Australie), Australian Government Publishing Service. 